\title{
Clinical homeopathic study in the homeopathic ambulatory at UNIRIO University Hospital, Brazil.
}

\section{Débora Alves dos Santos Fernandes, Francisco José de Freitas}

Federal University of the State of Rio de Janeiro - UNIRIO, Rio de Janeiro, Brazil

\begin{abstract}
Background: In 1966, the Gaffrée and Guinle University Hospital - GGUH was incorporated into the Federal University of the State of Rio de Janeiro - UNIRIO. Since then the Homeopathy clinic has had it's service there. Since the creation of medical residency in Homeopathy in 2004, there has been a significant increase of treatment in the number of patients and also a significant increase in the inter-relationship with other specialists and hospital services. A wide variety of patients have been treated at the GGUH on a daily basis. Many patients have been examined and treated with homeopathy alone, while others were also examined in other specialties, making homeopathic treatment an adjunct to classical therapy and vice versa. Given the expressive number of medical consultations (around 2500/year), this outstanding result in our clinical and academic practice has created the relevance and need for the verification of the epidemiological profile of these patients. Epidemiological studies are needed to clearly show the population included in this hospital, which is considered to be a reference in the Brazilian Health System (SUS).
\end{abstract}

Aims: To demonstrate the epidemiological and clinical homeopathic profile in the ambulatory of homeopathy at the Gaffree and Guinle University Hospital (GGUH) of the Federal University of the Staate of Rio de Janeiro - UNIRIO, Brazil.

Methodology: Cross-sectional study of 140 medical records randomly selected from the ambulatory of homeopathy at GGUH. The sample represents $50 \%$ of the monthly average of the clinical service from January to October 2009. The medical records incorrectly completed for the data analysis were excluded. Microsoft Office Excel 2007 used to data collection and analysis.

Results and Discussion: A total of 100 records were selected for analysis (71.4\%). Average age: 50 years old, $69 \%$ being between 41 and 80 years old. Gender: $79 \%$ women and 21\% men. Marital status: $46 \%$ married, 36\% single, $10 \%$ divorced and $8 \%$ widowed. Color: $61 \%$ white, $31 \%$ colored and $8 \%$ black. Education: Only $12 \%$ obtained an undergratuadion degree. Groups of diseases: Group-01 (48\%) high prevalence: $19 \%$ respiratory, $15 \%$ osteoarticular and $14 \%$ psychiatric; Group-02 (45\%) medium prevalence: $11 \%$ gastrointestinal, $9 \%$ cardiovascular, $8 \%$ genitourinary, $6 \%$ neurological, $6 \%$ dermatologic and $5 \%$ endocrine; Group-03 (7\%) low prevalence: $3 \%$ otological, $2 \%$ immunological, 1\% oncological and 1\% ophtalmological. $82 \%$ of the patients were treated solely with homeopathy and $18 \%$ with homeopathy and allopathy. Clinicalhomeopathic diagnosis: 53\% functional, 38\% lesional; $9 \%$ sensorial. Constitutional diagnoses: $31 \%$ sulfuric, $25 \%$ phosphoric, $22 \%$ carbonic, $22 \%$ mixed. Miasmatic / diathesic diagnosis: $47 \%$ sycotic, $28 \%$ psoric, $12.5 \%$ mixed, $9.5 \%$ tuberculinic, $3 \%$ syphilinic.

Conclusion: This study demonstrated a higher prevalence of women over 50 years old with respiratory, osteoarticular and psychiatric diseases. Most of them had a sulfuric constitution and presented functional diseases of sycotic origin. Homeopathy was the most adopted treatment during the period studied. This knowledge led to the creation of the "Homeopathic Clinical Identification Summary" which is used in the 
GGUH ambulatory. Epidemiological and clinical data were extracted from this summary with the objective to construct clinical trials.

Keywords: Epidemiological studies, homeopathy, prevalence, UNIRIO

\title{
References:
}

Cornillot P. Tratado de Homeopatia. Trad. Jeni Wolf. Porto Alegre: Editora Artmed, 2005.

Detinis L. Semiologia homeopática. Buenos Aires: Albatros, 1988.

Egito JL. Homeopatia Introdução è Teoria Miasmática. São Paulo: Robe Editorial, 1999.

Hahnemann CFS. Exposição da doutrina homeopática ou Organon da Arte de Curar (Organon der Heilkunst). Trad. de Eric Grüen. 2a ed. São Paulo: Grupo de Estudos Homeopáticos Benoit Mure, 1984.

Kleijnen J. Clinical trials of homoeopathy. BMJ 1991, 302: 316-23.

\section{Perfil Clínico Epidemiológico do Ambulatório de Homeopatia do Hospital Universitário da UNIRIO, Brasil.}

\author{
RESUMO
}

Introdução: A clínica de Homeopatia do Hospital Universitário Gaffrée e Guinle- HUGG existe desde 1966, quando o hospital foi incorporado à Universidade Federal do Estado do Rio de Janeiro - UNIRIO. Desde a criação da residência médica em Homeopatia em 2004, houve um aumento significativo no número de pacientes atendidos e na inter-relação com outros especialistas e serviços hospitalares. Dado o expressivo número de consultas médicas (cerca de 2500/ano), este excelente resultado em nossa prática clínica e acadêmica criou a relevância e necessidade para a verificação do perfil epidemiológico desses pacientes. Com a experiência de trabalho diário no HUGG, são observadas uma grande variedade de pacientes e doenças. Muitos pacientes são examinados e tratados pela homeopatia isoladamente, enquanto outros são vistos também em outras especialidades, fazendo tratamento homeopático como adjuvante à terapia clássica e viceversa. Estudos epidemiológicos são necessários para mostrar claramente a população incluída neste hospital, que é considerado referência no Sistema Único de Saúde (SUS).

Objetivos: Demonstrar o perfil epidemiológico e clínico homeopático do ambulatório de homeopatia do Hospital Universitário Gaffrée e Guinle (HUGG) da Universidade Federal do Rio de Janeiro Estado (UNIRIO), Brasil.

Metodologia: Estudo transversal de 140 registros médicos selecionados aleatoriamente no ambulatório de homeopatia no HUGG. A amostra representa 50\% da média mensal da assistência clínica, de janeiro a outubro de 2009. Os prontuários preenchidos incorretamente para a análise dos dados foram excluídos. O programa Microsoft Office Excel 2007 foi utilizado para a coleta e análise de dados. 
Resultados e Discussão: Um total de 100 registros foram selecionados para análise (71,4\%). Média de Idade: 50,18 anos de idade, sendo $69 \%$ entre 41 e 80 anos de idade. Sexo: as mulheres $79 \%$ homens e $21 \%$. Estado civil: $46 \%$ casados, $36 \%$ solteiros, $10 \%$ divorciados e $8 \%$ viúvos. Cor: $61 \%$ branco, $31 \%$ pardos e $8 \%$ negros. Apenas $12 \%$ obtiveram escolaridade segundo grau completo. Observou-se pacientes com três grupos de patologias: Grupo-01 (48\%) alta prevalência: 19\% respiratórias, 15\% osteoarticulares e 14\% psiquiátricas; Grupo-02 (45\%) média prevalência: 11\% gastrointestinal, 9\% cardiovascular, 8\% geniturinário, 6\% neurológicas, $6 \%$ dermatológicas e 5\% endócrinas; Grupo-03 (7\%) de baixa prevalência: 3\% otológica, $2 \%$ imunológicos, $1 \%$ oncológico e 1\% oftalmológicas. $82 \%$ dos pacientes foram tratados apenas com homeopatia e 18\% com a homeopatia e alopatia. Diagnósticos Clínico-homeopáticos: 53\% funcional, 38\% lesional; $9 \%$ sensorial. Diagnósticos constitucionais: $31 \%$ sulfúrico, $25 \%$ fosfórico, $22 \%$ carbônico, $22 \%$ mistas. Diagnósticos Miasmáticos/diatésicos: 47\% Sicóticos, 28\%psóricos, 12,5\% mistos, 9,5\% tuberculínicos, 3\% sifilinicos.

Conclusão: Este estudo demonstrou uma maior prevalência de mulheres acima de 50 anos de idade e de doenças respiratórias, doenças osteoarticulares e psiquiátricas. A maioria dos pacientes com diagnóstico constitucional eram sulfúrico e apresentavam doenças funcionais de origem sicótica. A homeopatia foi o tratamento mais adotado durante o período estudado. Este conhecimento levou à criação do Ficha de Identificação Clínico-Homeopática, que é usado atualmente no ambulatório do HUGG, e, a partir deste trabalho, os dados epidemiológicos e clínicos poderão ser extraídos com mais objetividade e praticidade e permitirá a construção de ensaios clínicos específicos.

Palavras-chave: Estudos epidemiológicos, ambulatório de Homeopatia, prevalência, UNIRIO.

\section{(cc) BY-NC-ND Licensed to GIRI}

Support: authors declare that this study received no funding

Conflict of interest: authors declare there is no conflict of interest

Correspondence author: Débora Alves dos Santos Fernandes - deboraunirio@yahoo.com.br , Francisco José de Freitas fjdefreitas@gmail.com

How to cite this article: Fernandes DAS, Freitas FJ. Clinical Homeopathic Study in the Homeopathic Ambulatory at UNIRIO University Hospital, Brazil. Int J High Dilution Res [online]. 2011 [cited YYYY Month dd]; 10(36): 203-205. Proceedings of the XXV GIRI Symposium and VIII CBFH; 2011 Sep 04-07; Foz do Iguaçu (Brazil). GIRI and ABFH; 2011; Available from: http://www.feg.unesp.br/ ojs/index.php/ijhdr/article/view/482/495 